\title{
COST-EFFECTIVENESS OF TREATMENTS FOR MILD-TO-MODERATE OBSTRUCTIVE SLEEP APNEA IN FRANCE
}

\section{Anne-Isabelle Poullié}

Haute Autorité de Santé

Magali Cognet

Amaris UK Ltd.

cognet.magali@gmail.com

Aline Gauthier, Marine Clementz, Sylvain Druais

Amaris UK Ltd.
Hans-Martin Späth

University Claude Bernard

Lionel Perrier

Cancer Centre Léon Bérard

Oliver Scemama, Catherine Rumeau Pichon, Jean-Luc Harousseau Haute Autorité de Santé

Objectives: Untreated obstructive sleep apnea-hypopnea syndrome (OSAHS) is associated with excessive daytime sleepiness, increased risk of cardiovascular (CV) disease, and road traffic accidents (RTAs), which impact survival and health-related quality of life. This study, funded by the French National Authority for Health (HAS), aimed to assess the cost-effectiveness of different treatments (i.e., continuous positive airway pressure [CPAP], dental devices, lifestyle advice, and no treatment) in patients with mild-to-moderate OSAHS in France.

Methods: A Markov model was developed to simulate the progression of two cohorts, stratified by CV risk, over a lifetime horizon. Daytime sleepiness and RTAs were taken into account for all patients while $\mathrm{CV}$ events were only considered for patients with high CV risk.

Results: For patients with low CV risk, incremental cost-effectiveness ratio (ICER) of dental devices versus no treatment varied between 32,976 EUR (moderate 0SAHS) and 45,579 EUR (mild OSAHS) per quality-adjusted life-year (QALY), and CPAP versus dental devices, above 256,000 EUR/QALY. For patients with high CV risk, CPAP was associated with a gain of 0.62 QALY compared with no treatment, resulting in an ICER of 10,128 EUR/QALY.

Conclusion: The analysis suggests that it is efficient to treat all OSAHS patients with high CV risk with CPAP and that dental devices are more efficient than CPAP for mild-to-moderate OSAHS with low CV risk. However, out-of-pocket costs are currently much higher for dental devices than for CPAP (i.e., 3,326 EUR versus 2,430 EUR) as orthodontic treatment is mainly non-refundable in France.

Keywords: Continuous positive airway pressure, Dental devices, Obstructive sleep apnea, Cost-effectiveness analysis

In France, obstructive sleep apnea-hypopnea syndrome (OSAHS) is associated with a major health and economic burden due the relatively high prevalence of the condition (i.e., 4 percent of the male adult population and 2 percent of the female adult population) and to the increasing use of medical devices such as continuous positive airway pressure (CPAP) $(1 ; 2)$. In January 2008, the French Ministry of Health requested a reevaluation of the medical devices (MD) included on the list of products and services approved for reimbursement (LPPR), for respiratory failure. Two commissions of the French National

This project was commissioned by the Ministry of Health, funded by the HAS and undertaken by Amaris. The technical report of this research (including the deterministic and probabilistic analyses) is available online at www.has-sante.fr. Many thanks to the 38 experts who provided critical advice and contribution for this project, and to the HAS (Clément V., Cohen-Akenine A., Couilleront-Peyrondet AL., Hamers F. and Ghabri S., Leclerc, S., Pessel C.) and Amaris colleagues (Doutriaux A., Jiang Y., Selya-Hammer C and Taieb V.) who worked on this analysis or publication and do not appear as co-authors.
Authority for Health (HAS) worked in parallel on this multiple technology assessment: (i) The National committee for the evaluation of medical devices and health technologies (CNEDiMTS), and its internal services, the Department of Assessment of Medical Devices (SED), assessed the clinical benefit of the MD in respiratory failure; and (ii) The Commission for Economic Assessment and Public Health (CEESP), and its internal services, the Health Economics and Public Health Department (SEESP), assessed specifically the cost-effectiveness of the MD in OSAHS.

OSAHS is a chronic condition characterized by the occurrence, during sleep, of successive episodes of cessation or decrease in respiratory airflow (2). Apnea and hypopnea are caused by repetitive collapse of the upper airways that may be either partial (hypopnea) or total (apnea). Symptoms have an important impact on health-related quality of life (HRQoL) and include excessive daytime somnolence, nonrefreshed sleep, nocturia, excessive snoring, choking during sleep, morning headaches, 
Poullié et al.

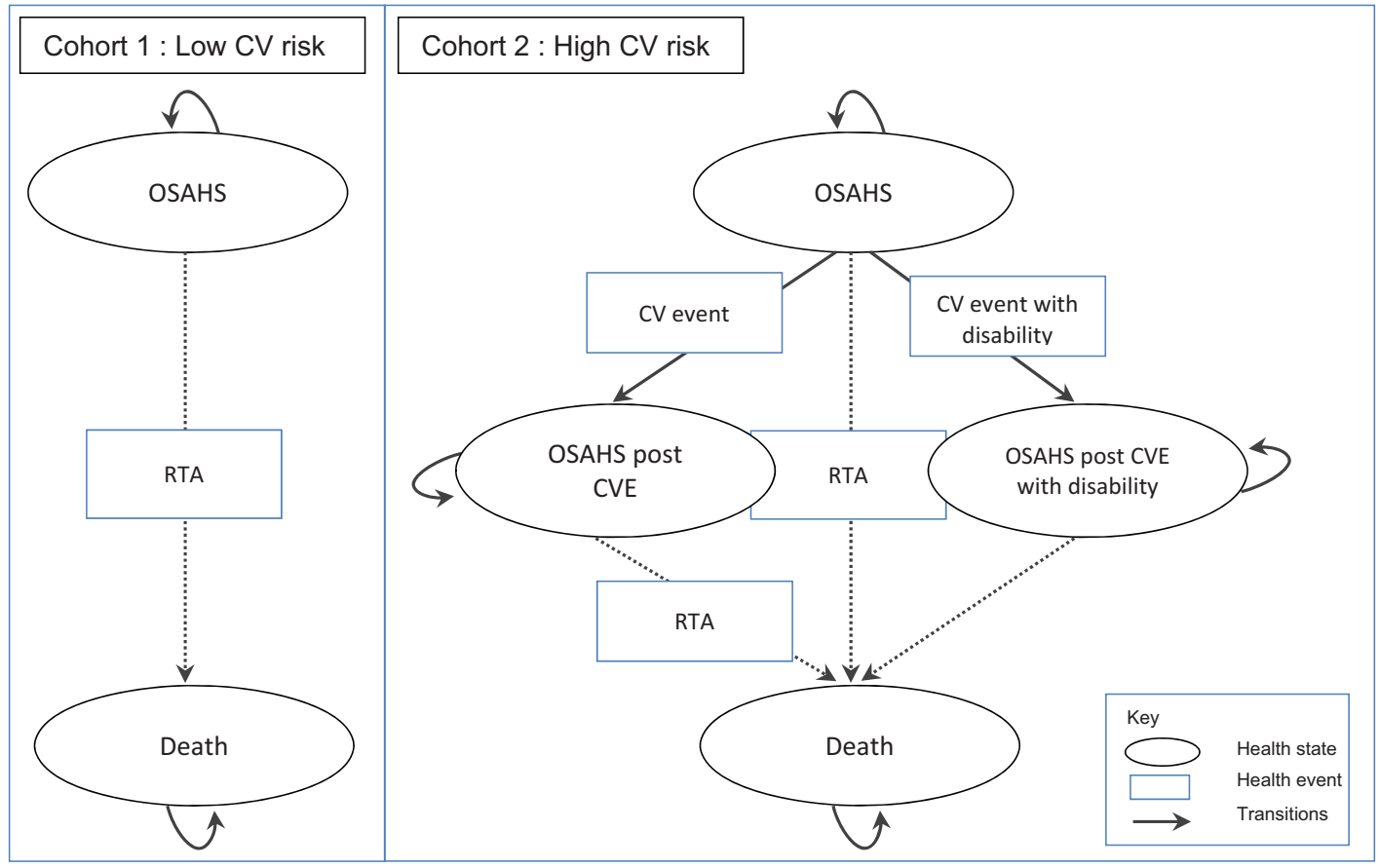

OSAHS: Obstructive sleep apnea-hypopnea syndrome; CVE: Cardiovascular event; RTA: Road traffic accidents

Figure 1. Structure of the Markov models.

and sexual dysfunction (2). If left untreated, OSAHS can lead to serious consequences such as increased risk of cardiovascular (CV) disease and road traffic accidents (RTAs).

Tests such as the apnea/hypopnea index (AHI) and the Epworth Sleepiness Scale (ESS) are widely used to diagnose OSAHS and to classify the severity of the condition $(2 ; 3)$. The AHI represents the number of apnea and hypopnea events per hour of sleep. The ESS uses a very short questionnaire and rates the daytime sleepiness between 0 (normal) and 24 (severe). In France, OSAHS severity relies partially on the AHI where mild is defines as an AHI between 5 and 15, moderate between 15 and 30 , and severe above $30(1 ; 2)$.

In the mild-to-moderate OSAHS population, treatment effect on CV events appears to be linked to patients' CV comorbidity. Indeed, observational studies conducted tend to show that CPAP reduces the incidence of $\mathrm{CV}$ only for patients with high CV comorbidities at baseline. Over a 10-year period, Marin et al. (4) followed over 1,000 patients with relatively few CV comorbidities (i.e., 35 percent of patients with hypertension, 11 percent with diabetes, 8 percent with hyperlipidemia, and 8 percent with $\mathrm{CV}$ disease). The number of events was similar between patients untreated and treated with CPAP. These results differ from those found in the study by Buchner et al. (5) who, over 6 years, followed 400 patients with a high level of CV comorbidity (i.e., 71 percent of patients with hypertensions, 23 percent with diabetes, 60 percent with hyperlipidemia, and 8 percent with other $\mathrm{CV}$ disease). The results showed that CPAP treatment significantly reduced the risk of $\mathrm{CV}$ events (estimated event-free survival after 10 years was 51.8 percent in untreated and 80.3 percent in treated patients; log-rank test, $p<.001$; absolute risk reduction, 28.5 percent). Campos-Rodriguez et al. (6) came to similar conclusions with their observational study among female patients with high levels of CV comorbidity.

There is a lack of cost-effectiveness evaluation of treatment options in patients with mild-to-moderate OSAHS. To our knowledge, only one previous study has been published by Weatherly et al. (7), which summarized the health technology assessment (HTA) conducted by McDaid et al. (3).

This study, funded by the HAS, aimed to assess the costeffectiveness of different treatments (i.e., CPAP, dental devices, lifestyle advice, and no treatment) for patients with mild-tomoderate OSAHS, with low or high CV comorbidities at baseline, in France.

\section{MATERIALS AND METHODS}

\section{Model Structure}

The model was developed using the HAS methodological guidelines (8) and a "collective perspective." This perspective takes into account all direct costs borne by all healthcare payers (including for instance complementary insurance and out of pocket costs); it does not include indirect costs. A Markov model was developed to simulate the progression of disease in mild-tomoderate adult patients with OSAHS (Fig. 1). As discussed in the introduction, in the mild-to-moderate OSAHS population, treatment effect appears to be partially linked to patients' CV 
comorbidity. Based on this evidence, the study population was stratified by CV risk $(4 ; 5)$. Two different cohorts were, therefore, considered, according to the level of $\mathrm{CV}$ risk at baseline, with two different corresponding model structures based on two or four health states: Cohort 1: Patients with mild or moderate OSAHS and low CV risk at baseline. Markov model with two health states (OSAHS and death) and one risk of event (RTA). All treatment strategies were compared (i.e., CPAP, dental devices, lifestyle advice, and no treatment) by assessing treatment effects on daytime sleepiness and RTAs. Cohort 2: Patients with mild or moderate OSAHS and high CV risk. Markov model with four health states (i.e., OSAHS, OSAHS post CV event, OSAHS post CV event with disability and death) and three risks of events (i.e., CV event, CV event with disability, and RTA). CPAP, dental devices, and the absence of treatment were compared by assessing treatment effects on daytime sleepiness, $\mathrm{CV}$ events, and RTAs.

Patients who became disabled following a CV event were assumed to face no further risk of RTA. Patients discontinuing treatment were progressing according to the transition probabilities associated with no treatment.

Costs and health effects, both in terms of quality-adjusted life-years (QALY) and life-years gained (LYG), associated with the interventions were assessed over a lifetime horizon given the chronic nature of OSAHS, and were discounted at 4 percent per year during the first 30 years and 2 percent per year thereafter.

\section{Inputs}

The data used to estimate the parameters of the model were obtained from systematic reviews and meta-analyses $(3 ; 9)$, observational studies $(5 ; 10 ; 11)$, modeling studies $(3 ; 7)$, and expert opinion. All inputs are available in Table 1.

Patient Characteristics. Patient characteristics at baseline were obtained from European cohort studies and the French national database "Système National Inter-régime de l'Assurance Maladie" (SNIIRAM) $(3 ; 5)$. The SNIIRAM gathers all data on reimbursed health expenditure at the national level. Fifty-five percent of patients in the model were considered to have a chronic long-term illness. These patients benefited from a 100 percent reimbursement rate for incurred health expenses when applicable, versus 60 percent to 70 percent for other patients.

Daytime Sleepiness. The effect of treatment on daytime sleepiness is reflected in the model by an improvement in HRQoL and lower risk of injury and death due to RTAs. Treatment effect on daytime sleepiness, assessed by a reduction of the ESS scores, was estimated based on meta-analyses $(3 ; 9)$.

Cardiovascular Events. The CPAP effect on $\mathrm{CV}$ events is reflected in the model by an incidence reduction, and therefore an improvement in HRQoL and lower risk of death. Lifetime CV risk and excess mortality were simulated from Kaplan Meier curves from the German study by Buchner et al. (5). The curves were then digitalized using the GetData software and a parametric survival function was estimated based on an exponential distribution. The breakdown of CV events observed in Buchner et al. (5) for patients regardless of OSAHS severity was applied to mild and moderate OSAHS patients and was assumed constant over time. Rate of debilitative stroke was taken from literature (20).

Road Iraffic Accidents. Baseline RTA risk was estimated from a French database (10) taking into account both the number of road fatalities and injuries in 2011 for the population over 45 years old (i.e., 1,038 killed, 7,382 hospitalized, 12,769 slightly injured including 85 percent, 79 percent, and 69 percent of whom were males, respectively) and the number of people in France with a driver's license (40.4 million people of whom 30 percent were aged 55 or older). In the model, the probability of being killed or injured was adjusted to patient characteristics (i.e., age and sex). Excess risk due to OSAHS was taken from a French observational study (11) and was applied to patients of all severity levels. The impact of CPAP on RTA was taken from a meta-analysis ( 3 ) and no studies were identified that assessed the impact of dental devices or lifestyle advice on RTA. Therefore, an adjusted odds ratio for dental devices and lifestyle advice compared with no treatment was estimated by applying the ratio of the treatment effects on ESS to the odds ratio for RTA.

Death. Crude death rates by age, sex, and cause of death were obtained from data published by the French Institute of Health and Medical Research (INSERM) for 2010 (12). This all-cause mortality includes disease-specific mortality in the general population; therefore, a correction was applied, according to the specific cohort.

Compliance. Compliance, defined as the long-term adherence to medical device, was derived for CPAP from a French database (SNIIRAM). Due to a lack of data on compliance with dental devices and with lifestyle advice, including the literature, compliance with these latter treatments was assumed to be equal to that of CPAP.

Resource Use and Cost Estimation. Total costs, in EUR 2013, were broken down by healthcare payer. The current study takes into account the cost of MD and associated services as well as other healthcare resources, including any health-care use due to $\mathrm{CV}$ events and RTAs. Resources associated with MD were obtained mainly from the twenty experts of the working group. CV events and RTAs resources were analyzed from French databases and a governmental report (10). Direct costs were estimated wherever possible on production factors, defined as the resources consumed (i.e., goods, services, and time) to produce the health care being studied. If not available, costs were estimated on reimbursement tariffs.

The cost of dental devices was estimated as the average of different types of devices sold in 2012 and weighted by each 
Poullié et al.

Table 1. Model Inputs

Input (measure)

Value

Ref

Patient characteristics at baseline

Age (years)

Sex (\% male)

ESS score : mild OSAHS

ESS score : moderate OSAHS

Treatment effect on daytime sleepiness (ESS score)

CPAP versus no treatment : Mild OSAHS

CPAP versus no treatment : Moderate OSAHS

CPAP versus dental devices : Mild OSAHS

CPAP versus dental devices: Moderate OSAHS

Lifestyle advice versus no treatment: Mild-to-moderate OSAHS

Monthly probability to have a CV event

Untreated patients

Patients on CPAP

Breakdown events

Coronary heart disease

stroke

death from CV event

Rate of debilitative stroke:

RTA

Excess risk due to OSAHS

Monthly probability to die from a RTA, if untreated OSAHS

Monthly probability to be injured from a RTA, if untreated OSAHS

(PAP effect (OR)

Dental device effect (OR)

56

86

7

13

\section{$-1.5$}

$-2.04$

$-0.2$

$-0.2$

$-0.31$

0.0043687

0.0019503

$65.80 \%$

$19.7 \%$

$14.50 \%$

$30.9 \%$

2.09

0.0000172

0.0002930

0.17

0.19

0.97

Crude death rates per 100000 population, per sex and age

All cause, between 45 and 95+ years oldFrance mainland, 2010 (Code CIM)

Cerebrovascular disease (Code CIM)

Ischemic heart disease (Code CIM)

Road traffic accident (Code CIM)

Compliance

Patients continuing treatment at 6 years $(\%)$

Healthcare resources used for CPAP (number per year)

Specialist visit, first year

Specialist visit, subsequent year

Polysomnography, first and subsequent year

Polygraphy, first year

Healthcare resources used for dental devices during its lifespan

Specialist visit, first year

Specialist visit, subsequent year

Dentist visit

Orthodontist visit

Polysomnography, first year

Polysomnography, subsequent year (number/year)

Polygraphy, first year

Polygraphy, subsequent year (number/year)

\section{Not applicable}

$160-169$

$120-125$

V01-V99

66

2

1

1 for $15 \%$ of patient

1 for $15 \%$ of patient

2

1

1

10

2 for $15 \%$ of patient

1 for $15 \%$ of patient

2 for $85 \%$ of patient

1 for $85 \%$ of patient

\section{5}

5

3

3

3

3

Assumption

3

9

5, Parametric survival function : Exponential distribution)

5, Parametric survival function : Exponential distribution)

5

5

5

20

11

3,10

$3,10,11$

3

Assumption (Calculated from ESS score)

Assumption (Calculated from ESS score)

12

12

12

12

SNIIRAM

Official journal

Official journal

Official journal and expert opinion

Official journal and expert opinion

Official journal and expert opinion

Official journal and expert opinion

Expert opinion

Expert opinion

Expert opinion

Expert opinion

Expert opinion

Expert opinion 
Table 1. Continued

\begin{tabular}{|c|c|c|}
\hline Input (measure) & Value & Ref \\
\hline \multicolumn{3}{|l|}{ Unit cost, EUR (health insurance reimbursement) } \\
\hline Fatal CV event costs & $5914(100 \%)$ & PMSI \\
\hline Non-fatal CV event costs & $5167(100 \%)$ & PMSI \\
\hline Monthly CV event cost over subsequent years & $263(100 \%)$ & Health insurance database \\
\hline Monthly CV event cost over subsequent years, in the event of disability & $329(100 \%)$ & Health insurance database \\
\hline Fatal and non-fatal RTA & $5,295(100 \%)$ & 10 \\
\hline CPAP & $21.25 /$ week $(60 \%)$ & Official journal \\
\hline Dental device & $337(60 \%)$ & Health insurance database \\
\hline Polysomnography & $181.53(100 \%)$ & Health insurance database \\
\hline Polygraphy & $119.31(100 \%)$ & Health insurance database \\
\hline Specialist visit & $26.19(70 \%)$ & SNIIRAM \\
\hline Orthodontist visit for dental device & $564(161)$ & Expert opinion \\
\hline Dentist consultation & $23(70 \%)$ & Health insurance database \\
\hline \multicolumn{3}{|l|}{ Utility } \\
\hline ESS & $0.892+$ baseline ESS *-0.01 & 3 \\
\hline Stroke & -0.145 & 14 \\
\hline Myocardial infarction or unstable angina & -0.051 & 14 \\
\hline RTA & 0.62 & 13 \\
\hline
\end{tabular}

OSAHS, obstructive sleep apnea-hypopnea syndrome; RTA, road traffic accident; CV, cardiovascular ; ESS : Epworth Sleepiness Scale ; CPAP : Continuous Positive Airway Pressure; PMSI, Le Programme de médicalisation des systèmes d'information; MCO, Médecine Chirurgie Obstétrique; ENCC, études nationales de coûts à méthodologie commune; SNIIRAM, Système national d'information inter-régimes de l'Assurance maladie.

model's annual volume sales. Other costs related to intervention management were based on expert opinion and/or derived from French databases (Table 1). The weighted average cost of a specialist visit was based on a SNIIRAM analysis which assessed the proportion of specialists who prescribe CPAP (i.e., 50 percent lung specialist, 45 percent general practitioner, 2 percent cardiologist, 2 percent otolaryngology, and 1 percent neurologist visit). Costs of lifestyle advice could not be assessed and therefore were not included in the cost-effectiveness analysis.

Fatal and nonfatal CV event costs were calculated from French hospital databases "Programme de Médicalisation du Système d'Information" (PMSI), and applied in each cycle of the model for fatal and nonfatal incident cases. When available, the cost of each diagnosis related group (DRG, or "Groupe Homogène de Malade" (GHM)) was derived from the average cost observed in France ("Etudes nationales de coûts à méthodologie commune" (ENCC), 2011). Hospital stays were differentiated by whether they occurred in a public or private establishment. The cost of a polysomnography (PSG) exam was applied following a nonfatal $\mathrm{CV}$ event based on expert opinion.

Management of patients over time following a CV event was based on data published by the French health insurance of patient having this chronic condition.
RTA cost was based on a governmental report (10), and was applied for any kind of RTA, regardless of its severity.

Utility. Excessive daytime sleepiness, RTA, CV disease, and death were considered while taking into account patient quality of life. Due to a lack of published French data on this topic, utilities used in the model were derived from European literature using EQ-5D questionnaires (13-15). The EQ-5D French set of preferences values could not be applied to these studies as patient level data were not available. Utility values used in the model are reported in Table 1.

The models estimating the relationship between ESS and HRQoL weights based on the EQ-5D derived by McDaid et al. (3) was used in our analysis. The authors derived an equation to estimate the utility value associated with daytime sleepiness due to OSAHS.

Data for CV events were obtained from a Swedish study by Lindgren et al. (14) who followed sixty mild and moderate hypertensive patients over 1 year in 2003 within the ASCOT clinical trial and estimated utility losses associated with experiencing the first $\mathrm{CV}$ event.

The utility value for RTA was obtained from Currie et al. (13), who used the EQ-5D questionnaire on fifty-six Welsh 


\begin{tabular}{|c|c|c|c|c|c|}
\hline & \multirow{2}{*}{$\begin{array}{l}\text { Adjusted total cost, EUR } \\
\text { (cost refundable by } \\
\text { health insurance/other) }\end{array}$} & \multicolumn{2}{|c|}{ Treatment effects } & \multicolumn{2}{|c|}{ ICERs } \\
\hline & & LYGs & Adjusted QALYS & $\begin{array}{l}\text { Cost-effectiveness } \\
\text { (EUR/LYG) }\end{array}$ & Cost-utility (EUR/QALY) \\
\hline \multicolumn{6}{|c|}{ Cohort 1 : Low CV risk } \\
\hline No treatment & $\begin{array}{l}307 \\
(307 / 0)\end{array}$ & 25.841 & $\begin{array}{l}\text { Mild: } 13.306 \\
\text { Moderate: } 12.335\end{array}$ & / & / \\
\hline Dental devices & $\begin{array}{l}7710 \\
(4384 / 3326)\end{array}$ & 25.865 & $\begin{array}{l}\text { Mild: } 13.469 \\
\text { Moderate: } 12.560\end{array}$ & 299103 & $\begin{array}{l}\text { Mild: } 45579 \\
\text { Moderate: } 32976\end{array}$ \\
\hline CPAP & $\begin{array}{l}13754 \\
(11324 / 2430)\end{array}$ & 25.866 & $\begin{array}{l}\text { Mild: } 13.493 \\
\text { Moderate: } 12.583\end{array}$ & 9108102 & $\begin{array}{l}\text { Mild: } 256048 \\
\text { Moderate: } 256278\end{array}$ \\
\hline \multicolumn{6}{|c|}{ Cohort 2 : High CV risk } \\
\hline No treatment & $\begin{array}{l}22652 \\
(22652 / 0)\end{array}$ & 25.072 & 11.997 & / & / \\
\hline CPAP & $\begin{array}{l}28980 \\
(26555 / 2425)\end{array}$ & 25.697 & 12.622 & 10119 & 10128 \\
\hline
\end{tabular}

CPAP, continuous positive airway pressure; CV, cardiovascular; ICER, incremental cost-effectiveness ratio; LYG, life-years gained; QALY, quality-adjusted life-year.

patients 6 weeks after hospitalization due to RTA. Values used in the model are detailed in Table 1.

\section{Analyses}

Due to the paucity of literature, incremental cost-effectiveness ratios (ICERs) for mild OSAHS and ICERs for moderate OSAHS could be calculated only for cohort 1 (low CV risk) when expressed in EUR/QALY. All other results are expressed for the pooled mild-to-moderate OSAHS population.

The robustness of results was assessed using deterministic (DSA) and probabilistic sensitivity analyses (PSA).

The parameters tested in DSA analysis were treatment effect and costs, health states utilities and costs, discount rate. Extreme case (worst- and best-case scenario) analyses were conducted as well as scenario analyses. For extreme scenarios, all utility values and probabilities of $\mathrm{CV}$ events were varied by \pm 10 percent, by 20 percent for cost of dental devices, by 25 percent for cost of CV and RTA and by 50 percent for treatment effect on daytime sleepiness. These variations were based on expert opinions. For the scenario analyses, a greater difference in treatment effect between dental devices and CPAP (-0.85 versus -0.2$)$ was applied (3). A separate DSA assumed a longer and more realistic 3-year lifespan of dental devices based on expert opinion, rather than 2 years, which is the legal time before reimbursement. Discount rates were varied in a subsequent DSA to 2.5 percent (16) and costs of $\mathrm{CV}$ events were increased by a factor of 2.3 compared with the base case to take into account the three PMSI databases: "Médecine Chirurgie Obstétrique" (MCO), "Soins de suite et de réadaptation" (SSR), and "Psychiatrie" (PSY) by means of a SNIIRAM analysis (17).

The PSA assesses the probability that each treatment represents the most cost-effective use of resources to be reported given currently available evidence. Uncertainty in probabilities and utilities were expressed in beta distributions, costs in gamma distributions, treatment effect on daytime sleepiness by a normal distribution, lifespan of dental device and adherence to medical devices by a uniform distribution.

\section{RESULTS}

\section{Base Case Analysis for Patients with Low CV Risk (Cohort l)}

For mild-to-moderate OSAHS patients with low CV risk: (i) Dental devices and CPAP offer similar treatment benefits (i.e., ESS, RTA risk, compliance) but the management cost associated with CPAP was 1.7 times higher than with dental devices. Total costs over lifetime were 7,710 EUR for Dental devices and 13,754 EUR for CPAP. Therefore, CPAP versus dental devices was associated with an ICER of 256,000 EUR per QALY and over 9 million EUR per LY gained (Table 2). (ii) ICERs of dental devices versus no treatment varied between 32,976 EUR/QALY (moderate OSAHS) and 45,579 EUR/QALY (mild OSAHS) and the cost per LYG was up to 300,000 EUR.

While dental device was identified as the optimal strategy from a cost-effectiveness standpoint, out-of-pocket costs were higher for patients (i.e., 3,326 EUR versus 2,430 EUR) due to mainly nonrefundable orthodontist care in France, including the application of the device. The part of the total cost that is refundable by health insurance is detailed in Table 2 . 


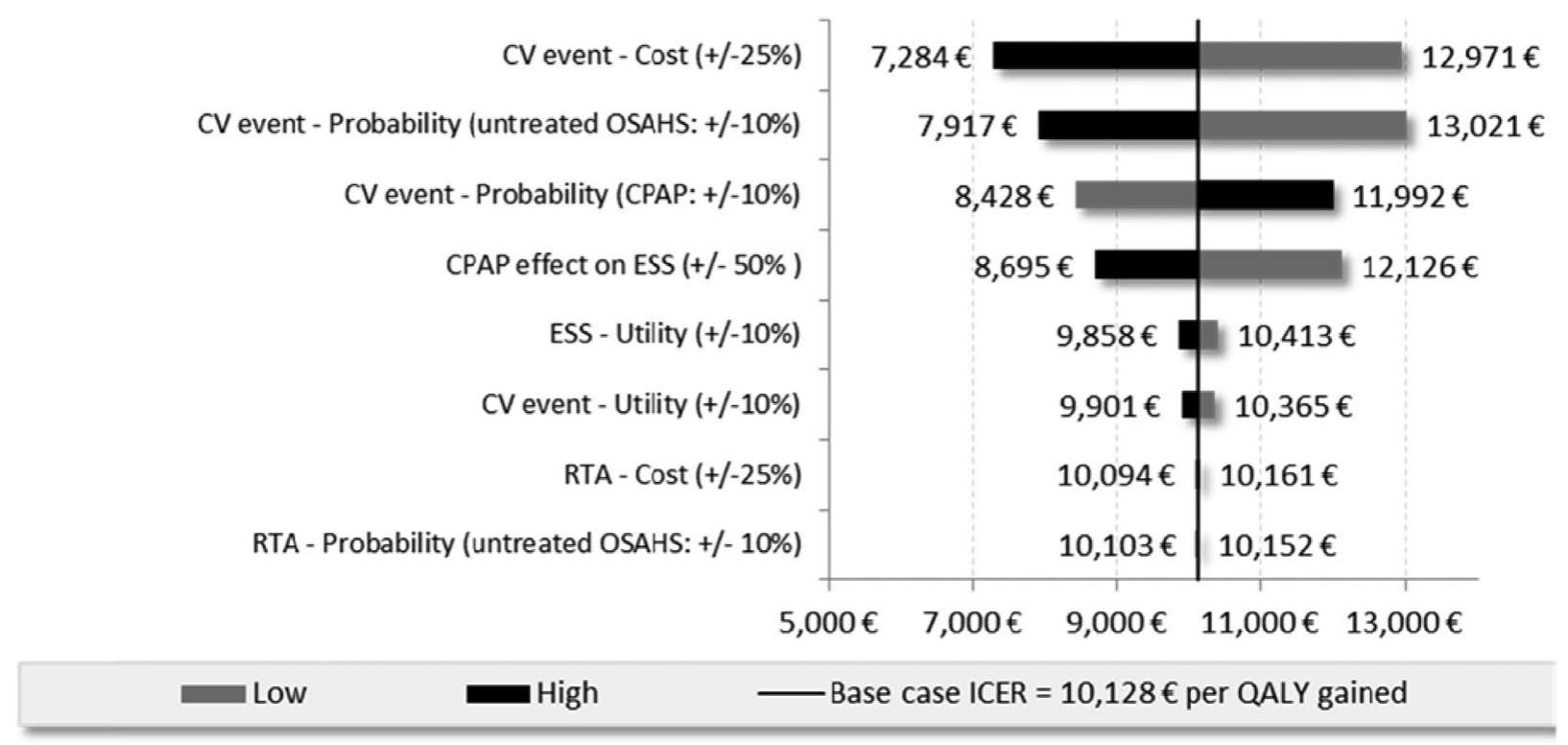

Figure 2. Tornado diagram of Cohort 2 (high CV risk), CPAP vs no treatment.

\section{Base Case Analysis for Patients with High CV Risk (Cohort 2)}

For mild-to-moderate OSAHS patients with high CV risk, CPAP was associated with an increase of 0.62 QALYs/LYGs compared with no treatment, resulting in ICERs of 10,128 EUR per QALY/LYG in both the cost-effectiveness and cost-utility analysis (Table 2). It is important to note that in the base case analysis, the treatment effect of CPAP versus no treatment was obtained directly from an observational study (2). The efficacy of dental devices on the prevention of $\mathrm{CV}$ events in mild-tomoderate OSAHS patients has not yet been published in the literature.

Sensitivity Analysis. According to the univariate sensitivity analyses, the key drivers of the ICERs were parameters related to sleepiness (i.e., treatment effect on ESS, utility related to ESS) and $\mathrm{CV}$ inputs (i.e., cost, probability, utility). The following tornado diagram (Figure 2) compares CPAP versus no treatment in patients with high $\mathrm{CV}$ risk at baseline (cohort 2).

For the moderate OSAHS population with low baseline CV risk, the ICER of dental devices versus no treatment was reduced by 26 percent when the product life of the medical device was extended by 1 additional year and increased by 25 percent when the treatment effect associated with dental devices on daytime sleepiness was significantly reduced $(24,525$ EUR/QALY and 41,065 EUR/QALY versus 32,976 EUR/QALY). Assuming a constant discount rate of 2.5 percent reduced the ICERs by 6 percent when comparing CPAP to no treatment for cohort 2 and less than 1 percent for all other situations. For the high CV risk population the ICER for CPAP versus no treatment resulted in a 14 percent reduction in costs of estimated CV costs based on three PMSI databases (MCO, HAD, PSY) with an ICER of 8,688 compared with 10,128 EUR/QALY.
The PSA showed that dental devices were the optimal strategy (versus CPAP and no treatment) for thresholds ranging from 30,000 EUR to 200,000 EUR in cohort 1. In cohort 2, CPAP (versus no treatment) reached full certainty of being the best strategy from 38,000 EUR/QALY threshold.

\section{DISCUSSION}

The objective of the current study was to assess the costeffectiveness for the different treatments for OSAHS among patients with mild-to-moderate severity in France. This costeffectiveness analysis together with the assessment of the clinical effectiveness of OSAHS treatments will support the French government in reimbursement decisions.

To our knowledge, this is the first cost-effectiveness analysis of treatment of mild-to-moderate OSAHS. Despite the uncertainty surrounding various input parameters mentioned above, this analysis clearly indicates conclusions for the two cohorts.

In France, it is efficient to treat all mild-to-moderate OSAHS patients with high CV risk with CPAP. Robust data for dental devices in this specific cohort was not available. By assuming an efficacy of dental devices up to 1.56 times less than that of CPAP on the prevention of $\mathrm{CV}$ events, the cost-effectiveness of dental devices would be the same as that for CPAP. Making an assumption of similar effectiveness based on an extrapolation of results reported by Anandam et al. (18), the costeffectiveness of dental devices becomes far superior to that of CPAP.

For mild-to-moderate OSAHS with low CV risk, dental devices are more efficient than CPAP. However, patient outof-pocket costs are currently much higher for dental devices than for CPAP because of mainly nonrefundable orthodontic 
treatment in France. Recommending orthodontic devices as first-line treatment for patients with mild-to-moderate OSAHS would require a consideration of the rate of reimbursement for costs associated with administration and fitting of the device.

The present analysis had several limitations. Due to a lack of published data in the literature on mild-to-moderate OSAHS, opinions of the 20 experts of the working group were requested on some inputs, especially level of resources used with CPAP and dental devices. Another limitation that is also present in other modeling studies is that sex difference on disease progression and treatment effects (i.e., impact on ESS score and $\mathrm{CV}$ events) could not be considered due to a lack of data on the topic. Costs of included outcomes (RTA and CV events) were likely underestimated, with treatment effect also underestimated, thus increasing the resulting ICERs. Few publications have documented the link between the incidence of diabetes and OSAHS, the extent to which diabetes is a risk factor or a complication of OSAHS, nor the treatment effect of OSAHS on diabetes. Fewer studies still have focused on the mild-to-moderate OSAHS population. As such, diabetes was not included in this health economic model. In the analysis of patients with low $\mathrm{CV}$ risk, the effectiveness of lifestyle advice could be evaluated without estimating the resource used to reach it, or the frequency of visits to general practitioners or nutritionists to ensure proper follow-up. To this date, there is no proper recommendation on duration for the management of OSAHS with lifestyle advices. Therefore, lifestyle advices could not be assessed in this costeffectiveness analysis.

A systematic literature review was conducted as a first step to develop the model structure comparing CPAP, dental devices, and no treatment, and neither model took into account long-term follow-up with a healthcare professional for lifestyle advices. As in other OSAHS models, the current analysis within the French context is based on a model which takes into account treatment effects on daytime sleepiness, RTA, and CV events. In contrast to models previously published in the literature $(3 ; 8 ; 15)$, the present analysis: (i) focuses on mild-to-moderate OSAHS in whom a treatment effect on $\mathrm{CV}$ events was only applied for patients with high baseline CV risk, and (ii) the incidence of $\mathrm{CV}$ events were modelled based on observational data from a German study (6) evaluating CPAP versus no treatment, rather than using risk equations based on variations of blood pressure (BP). This component of the model was bolstered by the study by Fava et al. (19) which demonstrated a lack of treatment effect on BP in a subgroup analysis of patients with mild-tomoderate OSAHS as opposed to patients with severe OSAHS. Additionally, the expert advisory board for this study highlighted that BP could not sufficiently explain CV risk related to OSAHS.

Only one other English study reported results by OSAHS severity, but only in their sensitivity analysis (3). For patients with mild OSAHS, the ICER associated with CPAP versus no treatment was $£ 20,585 / \mathrm{QALY}$ and $£ 9,391 / \mathrm{QALY}$ for mild and moderate OSAHS patients, respectively. In our costeffectiveness analysis, cohort 2 results (i.e., mild-to-moderate OSAHS with high CV risk) shows comparable results, displaying an ICER of 10,128 EUR/QALY for CPAP versus no treatment. In the base case analysis of the English study the authors obtained an ICER comparing CPAP with dental devices quite low (i.e., £4,335). These results are thus difficult to compare with those of the current French model given the populations are not the same, neither the integration of $\mathrm{CV}$ comorbidities nor the treatment effect on daytime sleepiness estimated by different methods (AHI or ESE).

This health economic evaluation and decision tool has highlighted the need to treat mild-to-moderate OSAHS patients with high CV risk with CPAP, and patients with low CV risk with dental devices. The distinct lack of clinical data available on the long-term efficacy of OSAHS treatments for mild-to-moderate patients, notably dental devices and lifestyle advice, highlights the need for further clinical studies to confirm the results of this modeling study.

\section{CONFLICTS OF INTEREST}

The authors declare that there are no conflicts of interest.

\section{REFERENCES}

1. Haute Autorité de Santé. Rapport SAHOS. Evaluation économique. September 2014. http://www.has-sante.fr/portail/upload/docs/ application/pdf/2014-09/rapport_sahos_-_evaluation_economique.pdf (accessed October 6, 2014).

2. Société de Pneumologie de Langue Française. Recommendations for clinical practice. Obstructive sleep apnea hypopnea syndrome in adults. Rev Mal Respir. 2010;27:806-833.

3. McDaid C, Griffin S, Weatherly H, et al. Continuous positive airway pressure devices for the treatment of obstructive sleep apnoea-hypopnoea syndrome: A systematic review and economic analysis. Health Technol Assess. 2009;13:iii-xiv, 1.

4. Marin JM, Carrizo SJ, Vicente E, et al. Long-term cardiovascular outcomes in men with obstructive sleep apnoea-hypopnoea with or without treatment with continuous positive airway pressure: An observational study. Lancet. 2005;365:1046-1053.

5. Buchner NJ, Sanner BM, Borgel J, et al. Continuous positive airway pressure treatment of mild to moderate obstructive sleep apnea reduces cardiovascular risk. Am J Respir Crit Care Med. 2007;176:1274-1280.

6. Campos-Rodriguez F, Martinez-Garcia MA, de la Cruz-Moron I, et al. Cardiovascular mortality in women with obstructive sleep apnea with or without continuous positive airway pressure treatment: A cohort study. Ann Intern Med. 2012;156:115-122.

7. Weatherly HL, Griffin SC, Mc Daid C, et al. An economic analysis of continuous positive airway pressure for the treatment of obstructive sleep apnea-hypopnea syndrome. Int J Technol Assess Health Care. 2009;25:26-34.

8. Haute Autorité de Santé. Choices in methods for economic evaluation. Saint-Denis, France: Haute Autorité de Santé; October 2012.

9. Thomasouli MA, Brady EM, Davies MJ, et al. The impact of diet and lifestyle management strategies for obstructive sleep apnoea in adults: A systematic review and meta-analysis of randomised controlled trials. Sleep Breath. 2013;17:925-935. 
10. Observatoire national interministériel de sécurité routière. La sécurité routière en France. Bilan de l'année 2011. Paris La Défense: Documentation Française; 2011.

11. Philip P, Sagaspe P, Lagarde E, et al. Sleep disorders and accidental risk in a large group of regular registered highway drivers. Sleep Med. 2010;11:973-979.

12. INSERM. Crude death rates per 100,000 population, France (mainland), 2010 http://www.cepidc.inserm.fr/cgi-bin/broker.exe (accessed September 2, 2013).

13. Currie CJ, McEwan P, Peters JR, et al. The routine collation of health outcomes data from hospital treated subjects in the Health Outcomes Data Repository (HODaR): Descriptive analysis from the first 20,000 subjects. Value Health. 2005;8:581-590.

14. Lindgren P, Kahan T, Poulter N, et al. Utility loss and indirect costs following cardio-vascular events in hypertensive patients: The ASCOT health economic substudy. Eur J Health Econ. 2007;8:25-30.

15. Sadatsafavi M, Marra CA, Ayas NT, et al. Cost-effectiveness of oral appliances in the treatment of obstructive sleep apnoea-hypopnoea. Sleep Breath. 2009;13:241-252.
16. Commissariat général à la stratégie et à la perspective. L'évaluation socio-économique des investissements publics. Tome 1. Paris: CGSP; 2013.

17. Tuppin P, Alexandre R, Drouin J, et al. Cout annuel pour l'assurance maladie de la prise en charge des AVC en France en 2011 d'après les données du système national d'information inter-régimes d'assurance maladie (SNIIRAM). Dans: 18e journée de la société française neuro-vasculaire. 20-22 novembre 2013, Paris: Maison International; 2013.

18. Anandam A, Patil M, Akinnusi M, et al. Cardio-vascular mortality in obstructive sleep apnea treated with continuous positive airway pressure or oral appliance: An observational study. Respirology. 2013;18:118490.

19. Fava C, Dorigoni S, Dalle Vedove F, et al. Effect of continuous positive airway pressure (CPAP) on blood pressure in patients with obstructive sleep apnea/hypopnea. A systematic review and meta-analysis. Chest. 2014;145:762-771.

20. Second European Stroke Prevention Study. ESPS-2 Working Group. $J$ Neurol. 1992;239:299-301. 